\title{
Research of Intersection Groups Optimization Control Method Based on Critical Path Identification
}

\author{
Ying Liu ${ }^{1, *}$, Fang $L^{1}$, and Jiayan Shen ${ }^{1}$ \\ ${ }^{1}$ Southeast University School of Transportation NO.2 Sipailou (210096), Nanjing, China
}

\begin{abstract}
An optimal intersection groups control method based on critical path identification is proposed. The control algorithm uses a binary search to gradually determine the scope of the intersection groups, uses the duality method to express the intersection groups, uses breadth-first search algorithm to solve the critical path, and finally uses the branch-and-bound method to solve the lane canalization, realizes the optimized output of phase-sequence and timing of intersection control signals. The control algorithm is realized using $\mathrm{C}++$ language, including the following functional modules: intersection range dynamic definition, critical path identification, space-time resource optimization, and online timing adjustment of signal timing parameters and so on. Finally, the control algorithm is verified by the actual road network of Changzhou City of China, the result shows that, the efficiency of traffic operation is significantly improved.
\end{abstract}

\section{Introduction}

The intersection groups of urban is a set of intersections of the city road networks that are geographically adjacent to each other, and there is a strong correlation [1]. Solving urban traffic congestion from the perspective of the intersection groups has been recognized by most scholars. In 1978, Turner I.F. and Shannon G.F. first mentioned the concept of intersection groups under few road interactions and applied it to cooperative control among three intersections [2]. Liu [3] (2011) studied and analyzed the process of interaction between traffic flow and intersections under the vehicle queuing variation of trunk road and control signals at intersections, based on this, a multi-lane oversaturated signal cooperative control system was proposed. Yang Xiaoguang first proposed the concept of intersection groups signal control in 2001[4] and conducted a systematic study of urban traffic congestion problems. Most scholars have carried out research on traffic conditions, optimization objectives, control strategies, and control methods, however, they did not consider the issue from the perspective of system design and software implementation [5-10]. This article constructed the intersection groups control system, optimized the algorithm of each subsystem, and finally realized the whole system using $\mathrm{C}++$.

\section{The framework of intersection groups priority control system}

The intersection groups optimization control system is divided into several modules: Intersection Groups scope division, critical path identification, space-time resource optimization, timing parameters online adjustment, data transmission. The specific control framework and data flow are shown in Figure 1.

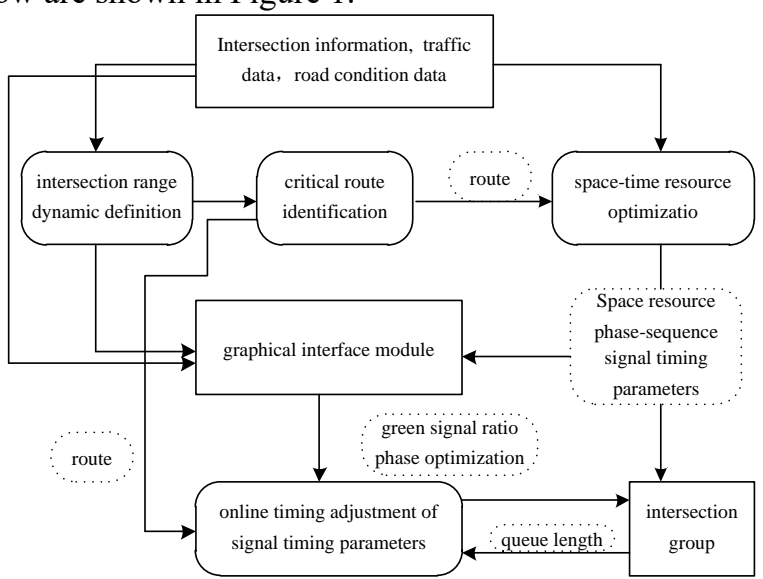

Figure 1. Optimization control of intersection control system

\subsection{Intersection groups scope division}

The intersection groups scope division module, firstly calculates the coordination control coefficient $\mathrm{CF}$ and the flow imbalance coefficient IF between the intersections based on traffic, period, average vehicle speed, and section length of intersections, and use the sum of CF and IF as the road segment correlation value. The self-organizing neural network adopts fuzzy means clustering algorithm to classify the strength of association. Using the branch-andbound method to gradually reduce the scale of the road networks to determine the signal control range of the intersection groups. 


\subsection{Critical route identification}

First, the junction map of the intersection groups is converted into a dual graph. The breadth-first search algorithm is used to search all the nodes in the dual graph, that is, all the road sections in the node graph. By comparing the ratio of the number of vehicles from the starting point to the end point, the degree of linkage discreteness index $I_{1}$ is calculated, and the degree of correlation between the length of the functional area and the distance of the road sections are used to calculate the degree of blocking-relevance index $\mathrm{I}_{2}$, and obtains the degree of path correlation I after dimensionless processing. By comparing the path association degree values of each path, the effective path with the largest path association degree value is determined as the key path of the current intersection groups.

The value of the path association degree $\mathrm{I}$ is:

$$
I=I_{1}^{\prime}+I_{2}^{\prime} \quad(I \in[0,2])
$$

\subsection{Space-Time resource optimization}

The mathematical programming method is used to realize the comprehensive optimization of time-space resources in intersection groups: the decision variables are represented by algebraic symbols, a binary mixed integer linear programming model is established according to the constraints and optimization objectives. The branch-andbound method is used to solve the canalization design, phase design and timing control parameter design scheme for each intersection of the intersection groups. There are nine phase-sequence options available after the design is implemented, one of which is shown in the figure.

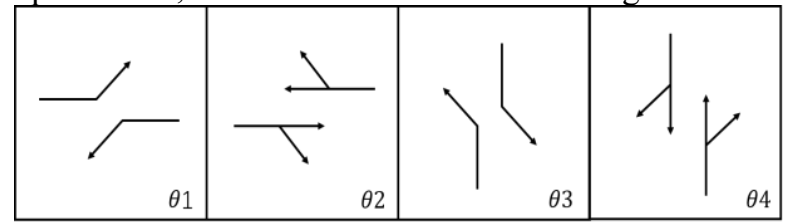

Figure 2. One of the phase-sequence options after time-space optimization design

\subsection{Signal timing parameters online adjustment}

Timing parameters online adjustment module consists of two sub-modules: green signal ratio adjustment module and phase difference optimization module.

The green signal ratio optimization module receives the real-time detection data, and the module continuously reads 10 pieces of data before the predetermined phase ends, each piece of data represents the detection data of each time unit in the detection period of 10 seconds. If extended green light time plus the minimum phase time is greater than the maximum green light duration, then the algorithm is extended to the maximum green light duration, and it switches to the next phase.

\section{Control system realization}

\subsection{Development platform}

The software development platform is selected as follows:

- Development language: $\mathrm{C}++$;

- Database management system: Oracle 9i or later.

- Operating System: Windows 7/8.

Performance requirements response time:

- Normal traffic status: $60-120$ s, oversaturated traffic status: $<240$ s;

- Control strategy conversion: $\leqslant 5 \mathrm{~min} ;$

- Intersection groups range change: $\leqslant 30 \mathrm{~min}$;

- Update processing time: $<120$ s;

- Data conversion and transmission time $:<1 \mathrm{~s}$;

- Optimization calculation time: $<30$ s.

\subsection{Data description}

The interface between independent modules uses data interface instead of Socket or other interface modes. The client program is designed based on the QT graphical interface library and provides a friendly man-machine interface. The database structure is shown in Table 1.

Table 1. Database structure

\begin{tabular}{|c|l|}
\hline \multirow{3}{*}{$\begin{array}{c}\text { Parameter } \\
\text { list }\end{array}$} & $\begin{array}{l}\text { left turn and straight travel equivalent } \\
\text { table, membership rating table of } \\
\text { detection time T, membership rating table } \\
\text { of accumulative arrival vehicle number A, } \\
\text { queuing intensity RP membership rating } \\
\text { table, first level fuzzy controller fuzzy } \\
\text { rule table, second level fuzzy controller } \\
\text { fuzzy rule table, green light Extended time } \\
\text { table }\end{array}$ \\
\hline Input list & $\begin{array}{l}\text { Intersection traffic information, physical } \\
\text { condition data table, road physical } \\
\text { condition data table, real-time data table } \\
\text { of detector }\end{array}$ \\
\hline Output & $\begin{array}{l}\text { critical path intersection sequence table, } \\
\text { control timing parameter table of critical } \\
\text { path intersection , real-time control state } \\
\text { table of signal light }\end{array}$ \\
\hline
\end{tabular}

\subsection{Graphical interface design}

The graphical interface module needs to display information: intersections input information, road section information, road network topology, and other information calculated by the software, such as target intersections, intersection groups, and critical paths. Figure 3 shows the software's main graphical interface. The left side shows the road network structure and the bottom map. In the figure, the solid dots in the figure represent the intersections, the red dots are the target intersections, the blue dots are the intersections within the intersection groups, and the red lines are the critical path. The right side of the figure indicates the intersection number, traffic capacity, phase sequence information and so on. The information of the corresponding intersection and road section can be displayed according to the selection taken by the mouse. 


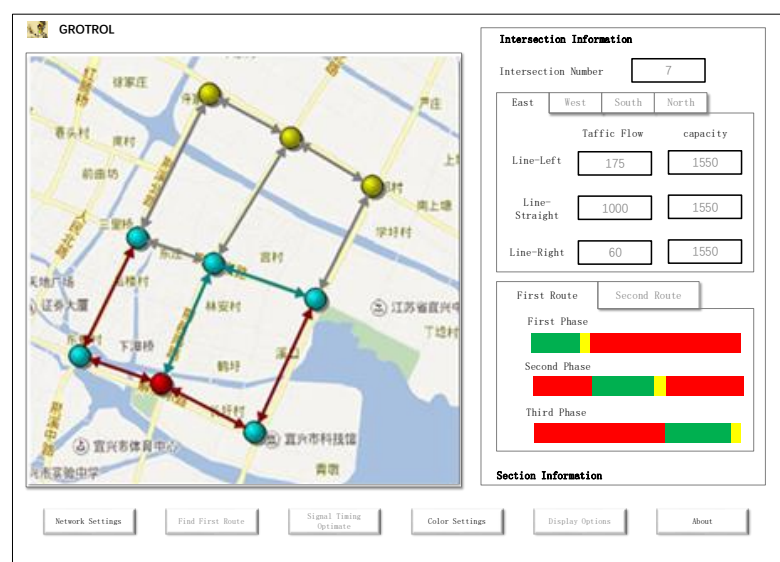

Figure 3. Graphical interface design of the optimization control system (In Chinese)

\section{Application analysis of the control system}

Take the intersection groups consisting of four intersections enclosed by Huxin Road, Nanqiao Road, Daoxiang Road, and Ziwei Middle Road of Quzhou City as an example. The data obtained from field investigation include traffic, control signal timing, travel speed, canalization, and length of the road section, etc. Figure 4 is a schematic diagram of a group of intersections. The letters on the road section indicate the direction of the entrance road, and the numbers indicate the intersection number. Among them, No.1, No.2, and No.4 intersections are respectively 3-way intersections, and No.3 intersections are 4-way type.

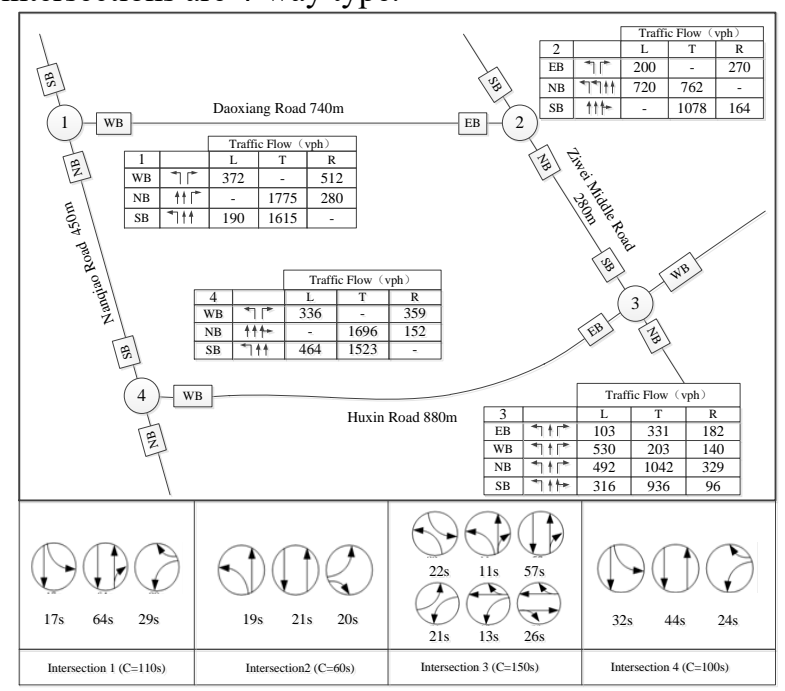

Figure 4. Layout of intersection groups

According to the above analysis method, the left-turn path of Daweixiang Road of Daoxiang Village is the critical path. Comparing the software designed in this paper with the Sychro, the results are shown in Figures 5 and 6 . The results show that, the proposed algorithm has the characteristics of smaller average delays and lesser average number of parking stops.

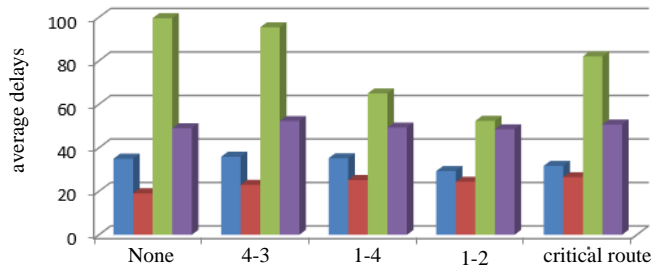

Figure 5. Traffic delays at all intersections

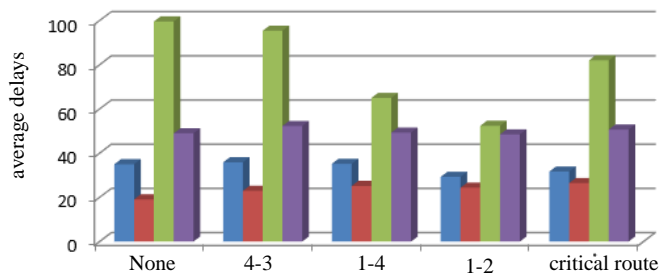

Figure 6. Average number of parking stops at each intersection

\section{Conclusion}

In this paper, an optimal control method for intersection groups based on critical path identification is proposed. The application results verify that, it can effectively improve the existing traffic operation status. The next step of the research is to add a bus priority control and special vehicle guidance module, to provide more reference for engineering practice.

\section{Acknowledgements}

This paper is sponsored by 'Universities Graduate Research and Innovation Plan of Jiangsu Province (No. CXLX_0141).

\section{References}

1. Li Yan. Research on Critical Path Identification and Traffic Signal Control of Oversaturated Intersections [D]. Nanjing: School of Transportation, Southeast University, 2011

2. Turner I F,Shannon G F. Micro master-the coordination of small groups of intersections[J].Institute of Transportation Engineers,1978,19(4):171-173.

3. Liu Y,Chang G L. An arterial signal optimization model for intersections experiencing queue spillback and lane blockage[J].Transportaion Research Part C: Emerging Technologies,2011,19(1):130-144.

4. Gao Yunfeng, Hu Huai, Han Hao,etc.Multi-objective optimization and simulation for urbanroad intersection groups signal control [J]. China Journal of Highway and traffic . 2012, 25(06):129-135

5. Li Yan, Guo Xoucheng, Yang Jie, Liu Ying, etc.Mechanism analysis and Implementation Framework for traffic signal control of over-saturated intersection groups [J].Journal of transportation systems engineering and information technology . 2011(04):28-34 
6. YangJie, Guo Xiucheng, Li Yan,etc.Modeling rout correlation degree of urban signed intersection groups [J]. Journal of transportation systems engineering and information technology. 2012(12):55-62

7. Yang Jie, Guo Xiucheng, Liu Ying, etc.Dynamic scope identification for urban intersection groups with traffic coordinate control [J]. Journal of transportation systems engineering and information technology. 2014(14):28-33

8. Li Yan, Guo Xiucheng, Yang Jie, etc. Routes classification methods atintersec [J]. Journal of Southeast University (Nature science edition ). 2012(42):168-172

9. Fu Hui, Xu Jianmin, Lu Kai. Signal control strategies for related group intersections based on particle swarm optimization [J]. Traffic and computer. 2007(25):23-26.

10. Hu Xinghua. Research on the optimization of bus priority control at signalize intersections based on cooperative vehicle infrastructure system [D]. BeiJing :BeiJing Jiaotong University , 2016. 